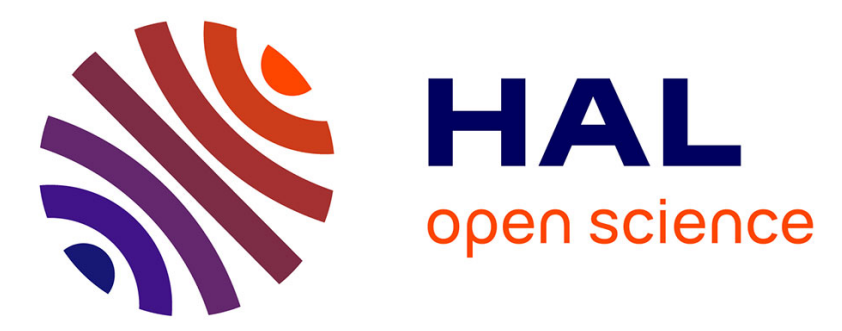

\title{
DET2007 Special Issue: The Representation of Manufacturing Requirements in Model-Driven Parts Manufacturing
}

\author{
Victoria Rogstrand, Torsten Kjellberg
}

\section{To cite this version:}

Victoria Rogstrand, Torsten Kjellberg. DET2007 Special Issue: The Representation of Manufacturing Requirements in Model-Driven Parts Manufacturing. International Journal of Computer Integrated Manufacturing, 2009, 22 (11), pp.1065-1072. 10.1080/09511920902741117 . hal-00528091

\section{HAL Id: hal-00528091 \\ https://hal.science/hal-00528091}

Submitted on 21 Oct 2010

HAL is a multi-disciplinary open access archive for the deposit and dissemination of scientific research documents, whether they are published or not. The documents may come from teaching and research institutions in France or abroad, or from public or private research centers.
L'archive ouverte pluridisciplinaire HAL, est destinée au dépôt et à la diffusion de documents scientifiques de niveau recherche, publiés ou non, émanant des établissements d'enseignement et de recherche français ou étrangers, des laboratoires publics ou privés. 


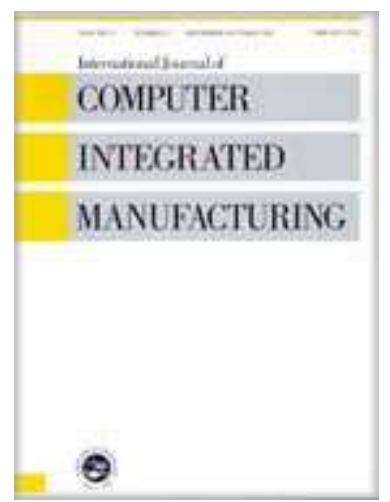

\section{DET2007 Special Issue: The Representation of Manufacturing Requirements in Model-Driven Parts Manufacturing}

\begin{tabular}{|c|c|}
\hline Journal: & International Journal of Computer Integrated Manufacturing \\
\hline Manuscript ID: & TCIM-2008-IJCIM-0044.R3 \\
\hline Manuscript Type: & Special Issue Paper \\
\hline $\begin{array}{r}\text { Date Submitted by the } \\
\text { Author: }\end{array}$ & 17-Nov-2008 \\
\hline Complete List of Authors: & $\begin{array}{l}\text { Rogstrand, Victoria; Royal Institute of Technology, Production } \\
\text { Engineering } \\
\text { Kjellberg, Torsten; Royal Institute of Technology, Production } \\
\text { Engineering }\end{array}$ \\
\hline Keywords: & INFORMATION, MANUFACTURING INFORMATION SYSTEMS \\
\hline Keywords (user): & Manufacturing, Requirements \\
\hline
\end{tabular}

\section{S ScholaroNE \\ Manuscript Central}




\section{The Representation of Manufacturing Requirements in Model-Driven Parts Manufacturing}

Victoria Rogstrand

Royal Institute of Technology, Production Engineering

Brinellvägen 66, SE-10044 STOCKHOLM, SWEDEN

Tel: +4687909078

Fax: +468210851

victoria.rogstrand@iip.kth.se

Corresponding Author

Prof. Torsten Kjellberg

Royal Institute of Technology, Production Engineering

Brinellvägen 66, SE-10044 STOCKHOLM, SWEDEN

Tel: +4687909069

Fax: +468210851

torsten.kjellberg@iip.kth.se 


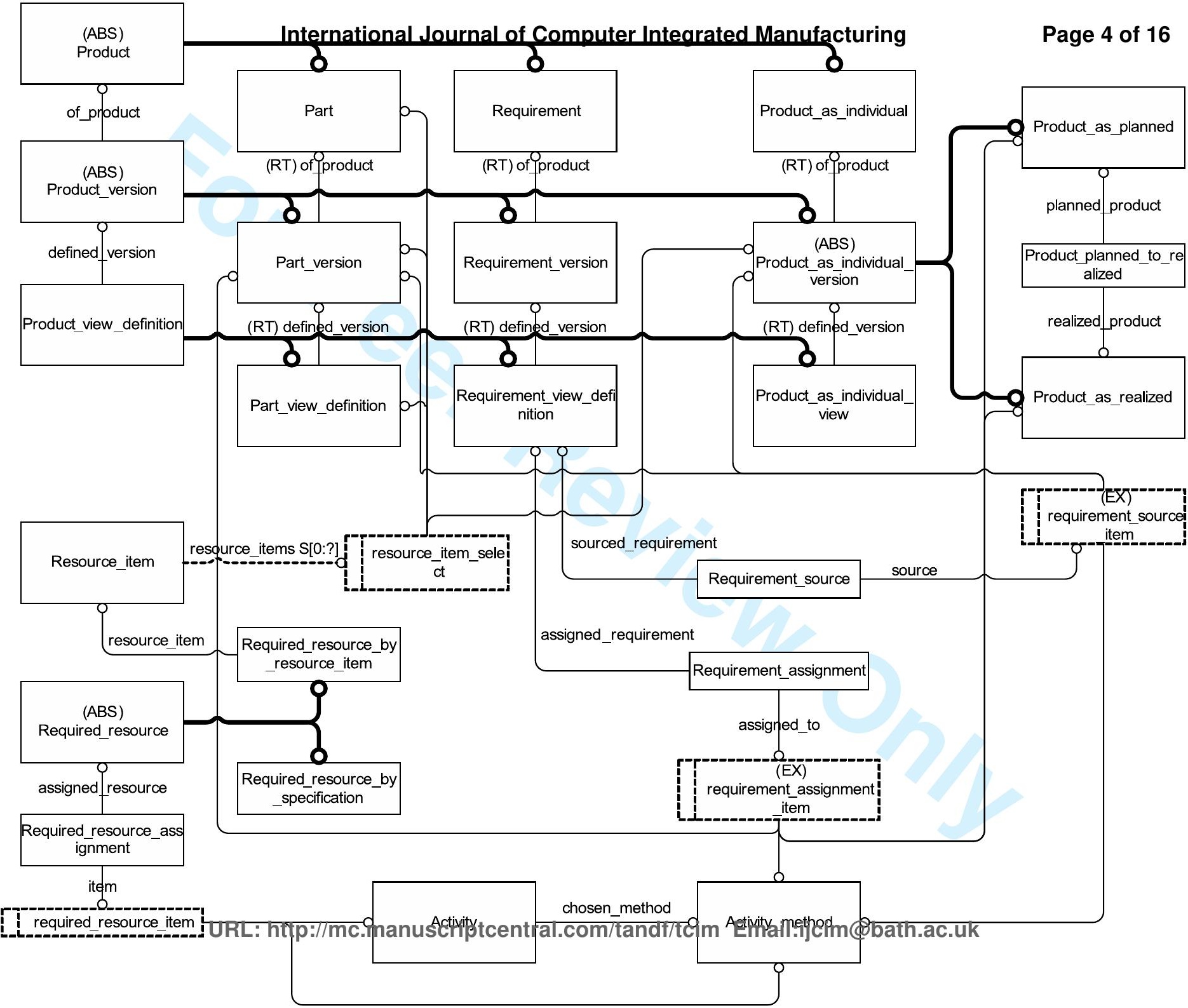




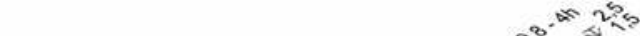

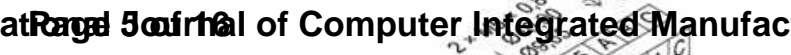
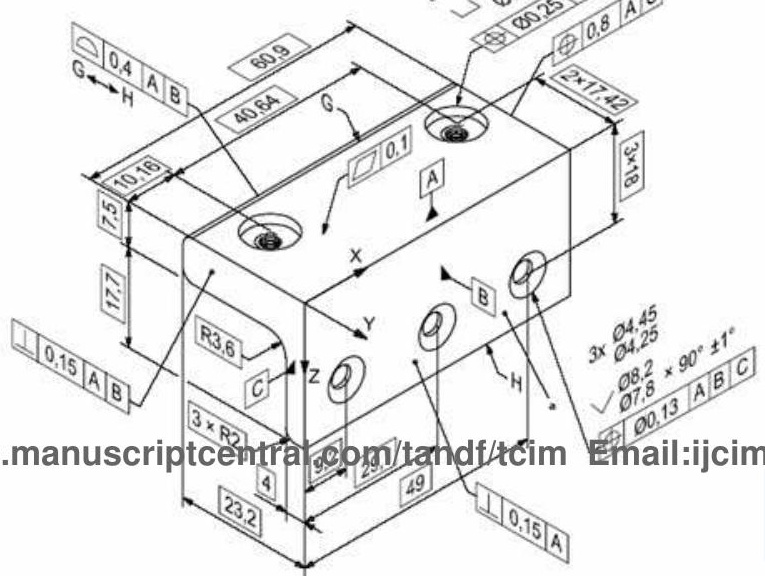


\section{The Representation of Manufacturing Requirements in Model-Driven Parts Manufacturing}

Victoria Rogstrand $^{1}$ and Torsten Kjellberg

Dept. Production Engineering, KTH Royal Institute of Technology, Stockholm, Sweden

Today there is a need to make process and production planning more cost-effective while not compromising the quality of the product. Manufacturing requirements are used to ensure producibility in early development phases and also as a source for continuous improvement of the manufacturing system. To make this possible it's essential to have correct, updated information available and to be able to trace the relations between requirements and their origin and subjects. To trace requirements origin in resources or processes is today very difficult due to system integration problems. This article discusses the relations that need to be represented and propose the use of model-based methods to enable traceability of requirements. Since requirements are a collaborative effort a standard for information exchange is needed. The ISO10303 STEP application protocol AP233 System Engineering is proposed for this purpose.

Keywords: Information Management; Manufacturing; Requirements

\section{Introduction}

One of the biggest challenges in early development phases of a product is the management of requirements. (Kerr, Roy, \& Sackett, 2006) A great effort is put on maintaining and managing requirements. The requirements must be attainable; stated in an unambiguous way; consistent, meaning that they do not contradict each other; and in the end verifiably to ensure the fulfilment of requirements (Harwell, Aslaksen, Hooks, Mengot, \& Ptack, 1993; Gilb, 1997). In addition to this there is the complexity of requirements' relationships; in collaborative development environments, requirements will originate from different domains and state different stakeholders' needs (Rogstrand \& Kjellberg, 2004). Manufacturing is one of the stakeholders (INCOSE, 2004). In the era of cutting time-to-market at lower cost, manufacturing systems' lifespan exceeds the product life on the market. Therefore, companies must meet the challenge of reusing existing manufacturing systems. This has led to a desire to secure the producibility of new products in the available manufacturing system through the use of requirements in early development phases, making the stakeholder manufacturing even more important. These requirements (henceforth referred to as manufacturing requirements) are complex, since some act on the product and originate from manufacturing while others act on the production itself (more thoroughly explained in Section 3 Representation of Requirements - Results of the Case Study).

Most requirements are still text-based meaning that they are defined as sentences in documents or systems. The management of requirements is therefore mainly handled through manual editing of documents. The large number of requirements makes the requirement documents endless and requirement management and

\footnotetext{
${ }^{1}$ Corresponding author. victoria.rogstrand@iip.kth.se
} 
verification, time-consuming and difficult, described by (Rogstrand \& Kjellberg, 2004; Nilsson, 2004; Kerr, Roy, \& Sackett, 2006; Melchisedech, 1998).

Therefore, more effort is now being exerted on management of requirements by dynamic methods that facilitate updating, derivation, decomposition, verification, etc. of requirements. This has led to an increasing use of requirement management tools to manage manufacturing requirements. Requirements management tools facilitate access, structuring and derivation of requirements (Nilsson, 2004).

However, existing commercial requirement management tools mostly support text-based requirements. They are often poorly integrated with data in other systems e.g. PLM-data (Bailey, 2004; Kerr, Roy, \& Sackett, 2006). Together this makes the process of verifying the requirements difficult and time-consuming. (Rogstrand \& Kjellberg, 2004) have showed that the poor support and variety of data, makes it necessary to be an area expert in order to be able to understand and verify the requirements. The importance of all stakeholders being able to understand the requirements have also been emphasised by (Lang \& Duggan, 2001).

Furthermore, in the case of manufacturing requirements there is also the issue that manufacturing requirements often secure producibility in an actual manufacturing system that is changing through time due to normal wear, maintenance and upgrades. The information about the manufacturing system and its processes is created and maintained in a variety of systems. The information is at different levels of details and the nomenclature of different domains varies a lot. Therefore, requirements from different stakeholders are neither consistent, nor homogenous, during the product development process. (Kerr, Roy, \& Sackett, 2006)

The objective of the research was to determine the information need of manufacturing requirements. Another objective was to examine what support is available for model-driven information representation of manufacturing requirements.

\section{Method}

A case study was performed with the aim of investigating the information needs for robust information management of requirements. Robust information is in this research article defined as updated, accurate information which is always available and interpretable by all concerned systems (Rogstrand \& Kjellberg, 2005). Based on the needs for a representation of manufacturing requirements, a study of the information standard ISO 10303, STEP, STandard for the Exchange of Product model data, has been conducted to evaluate if STEP may represent the necessary information about manufacturing requirements identified by the case study.

\subsection{The Case Study}

The case study was performed at a large truck manufacturer in Sweden to identify manufacturing requirements during process planning. To provide multiple sources of evidence, (Yin, 1990), both interviews and documents were used to collect data. The interviews were open-ended and focused (Yin, 1990). There were a mix of questions concerning facts and opinions of the interviewees. The interviewees were engineers with more than ten years experience. The language used was Swedish and the interviews were $1.5-2$ hours long. The data was collected during the winter of 2007/2008. The analysis of the interviews was made through 
explanation-building (Yin, 1990). Between every set of interviews careful analysis was done to evaluate the stated hypothesis. The collected documents (tenders from suppliers of manufacturing equipment) were developed during the purchase and process planning of a new manufacturing line in the plant, and contain information about manufacturing resources and processes. They also contain requirement information connected to the manufacturing line and its processes.

\subsection{Evaluation}

This part of the study evaluated how well the standard ISO 10303, STEP, STandard for the Exchange of Product model data, supports the information needs identified in the case study.

\section{Representation of Requirements - Results of the Case Study}

Requirements are often defined as functional requirements of a product, henceforth referred to as product requirements. Product requirements are conceptual and always act on the product. Conceptual objects are here defined as objects that are not yet present in the real world, e.g. the product during the early development phase or a new planned resource. Requirements may be derived from higher level requirements such as goals of quality, cost or time efficiency, which are then decomposed to form the product. In System Engineering, the V-model (INCOSE, 2004; Stevens, Jackson, Brook, \& Arnold, 1998) defines how every requirement has a solution at every detailing-level.

Conflicting requirements are balanced against each other to ensure that high priority requirements from all domains are fulfilled. During this process it is essential to have access to information about the origin of the requirements to evaluate consequences of non-fulfilled requirements. Decision-making is supported when the requirements of all domains can be balanced and evaluated against each other.

\subsection{Development of Requirement Representation}

The study confirms that requirements are often still text-based which makes the management of requirements a manual process of editing and managing documents. There are many problems related to this way of managing requirement information. Text-based requirements are time-consuming and error prone, since they must be manually edited and verified. This has led to the development and use of property-based requirements. (Micouin, 2008) A property-based requirement is a quantified representation of a requirement that is possible to evaluate using automated methods.

However, property-based requirements must still be manually defined. The quantification of a requirement is seen by many as a difficult process, which also means that the acceptance of the method is low. The fact that the quantification of a requirement may be difficult should not be seen as a problem. By trying to avoid the difficulty and using text-based requirements, the problem is only passed along downstream in the development process. It is the people that verify if a requirement is fulfilled who will suffer when the definition of the requirement is poorly done.

There are still problems with tracing the design solutions of manufacturing requirements, to ensure fulfilment of the requirements, but first and foremost of tracing the origin of manufacturing requirements and thereby evaluating consequences of non-fulfilled requirements. 


\subsection{Manufacturing Requirements}

Manufacturing Requirements are most often considered as limitations since they may limit the product design, e.g. Design For Manufacturing (DFM) where the choice of machining equipment constrains the machining process, and in turn also limits the product design. (Ulrich \& Eppinger, 2000) DFM is used to secure robust manufacturing. (Selvaraj, Radhakrishnan, \& Adithan, 2008) For example, if the existing manufacturing system is not capable of producing the specified features of the product, it means that the existing system puts a requirement, in the form of a limitation, on the design to secure the quality of the product. The manufacturing requirement states which one of a predefined set of processes that shall be used to ensure the quality of the product. The defined processes are known to produce quality based on the knowledge or strategy of the company. The use of a predefined set of processes also implies the use and maintenance of a process library. However, even though manufacturing requirements are not functional requirements but limitations of the product, they secure the quality of the product produced. Therefore, these requirements are not only limitations to ensure producibility, but may also be traced back to customer demands.

Manufacturing requirements also secure production logistic, ergonomic processes, reliable equipment, and ensures that manufacturing knowledge in the company is utilized. Manufacturing requirements ensure that best practice is used and that the product realization process is continuously improved. The improvement of the product realization process is achieved when recurring conflicts and non-fulfilments of requirements are highlighted and can be corrected. Although a manufacturing requirement may often limit the product, a major change e.g. due to a paradigm shift in the product, process or resource, must always be allowed. But, by knowing the constraints of the manufacturing system and its processes, the information can be used to make the right decisions. When it is time for investment in new manufacturing equipment, the current limitations are known and thereby also what part of the manufacturing system is in need of improvement, how to improve and how to specify the new equipment.

In the same way as product requirements, manufacturing requirements may be derivations of high level requirements. Manufacturing requirements can also be standards or environmental laws that need to be followed. The traceability of a requirement is a key issue in requirement management (Kirkman, 1998). The difference in comparison to product requirements is that manufacturing requirements are derived not only from other requirements, but can originate from the resources and processes being used to manufacture cf. Figure 1. The resources constrain the processes that can be performed. For example, a geometry limitation of the product may originate in the manufacturing equipments capability in positioning a cutting tool in a certain set up. They can also originate from the relationship between a process and a resource, or a product and a process, cf. Figure 1. This is an important aspect, since it might not be the process or resource themselves that are the source for a requirement, but rather the combination of the two, e.g. when a machine performs a certain process

Figure 1 Manufacturing requirements origin in product and manufacturing lifecycle data.

Manufacturing requirements may also be traced back to a product. Since several different products are being manufactured in the same manufacturing system, the products that are already being manufactured will limit the new products that may be introduced in order not to disrupt the manufacturing of the current products. This is however more common in assembly lines than in parts manufacturing.

Manufacturing requirements do not just act on the product as in the case of DFM, but on the manufacturing itself, its resources and processes, cf. Figure 2 
Figure 2 Manufacturing requirements.

\section{Evaluation of Manufacturing Requirement Representation in STEP}

Since the engineering process is a collaborative effort, there is a need to share requirement information. Requirements are communicated between different domains that use different information systems. During balancing of requirements from different domains it is essential to be able to evaluate the impact of a change during process planning. There is a need for robust information that gives a holistic view of the product realisation process. Robust information is in this research article defined as updated, accurate information which is always available and interpretable by all concerned systems. To ensure robust information, one common system-independent information platform is needed as explained by (Rogstrand \& Kjellberg, 2005). This means that all product information is managed in one logical information base and that relationships between different domains of information are maintained. This will ensure that the information is always up to date and that all systems have access to the same information.

The holistic view of the product realisation process enables consequence analysis of decisions. To fully be able to evaluate manufacturing consequences of a change in product or manufacturing system there is a need for representation of the information of the manufacturing during its whole lifecycle. There is also a need for versioning of this data as it is essential to be able to look back on history in how the manufacturing requirements and manufacturing system and its processes has changed over time.

Therefore, it makes sense to communicate and represent requirements through the use of standardized information models. STEP was chosen because it covers the relevant areas, is an international standard for product data, and has proven its usefulness in both research and industry. Since there are several stakeholders it is also important to have bidirectional traceability. During balancing of requirements, all requirements can't be met. It's then essential to know who owns the requirement to be able to evaluate consequences of nonfulfilled requirements. This is equally important for product requirements acting on the manufacturing, as well as manufacturing requirements acting on the product, hence the importance of bidirectional traceability.

\subsection{Representation of Manufacturing Life-cycle Data in STEP}

The difference between a product and a resource is simply the point of view. A product for one actor is a resource for another. Manufacturing resources are products that are in its 'in use'-phase. The STEP Application Protocol (AP) 239 Product Life Cycle Support (PLCS) (ISO 10303-239, 2004), which support the entire life-cycle of a product, is therefore a good match to use as a starting point

The resources and processes may be planned for a new product, but they often already exist in the existing manufacturing system. The existing manufacturing system contains what is here referred to as physical resources, actual physical manufacturing equipment that you can touch and that are positioned on the shop floor. Therefore it is essential to be able to handle data of real existing equipment and its processes as well as planned.

AP239 PLCS can represent information about the design of a product (part), and the realized product (product_as_individual) (ISO 10303-1164, 2004), as stated previously the difference between a product and a resource is simply the point of view, hence the same representation used for products can be used to describe 
resources (part and product_as_individual) (Johansson, 2001; Nielsen, 2003; Nyqvist, 2008). AP239 PLCS can also represent information about a planned process (activity) and an actual performed process (activity_actual) (Eurostep, 2008).

These aspects of AP239 PLCS make it a good starting point for a manufacturing life-cycle representation.

However, there are certain aspects of the way processes can be represented in AP239 PLCS that requires further investigation. In the context described in this research article, the representation required for a process may go beyond what is currently possible in AP239 PLCS.

\subsection{Representation of Manufacturing Requirements in STEP}

AP233 System Engineering (ISO CD 10303-233, 2007) manages requirements and different breakdowns during development of a system (Ratchev, Pawar, Urwin, \& Dirk, 2005) and focuses on handling requirements of all stakeholders of the product as described by (INCOSE, 2004). Production planning and preparation, and manufacturing are two of the stakeholders. Manufacturing requirements, as stated earlier in Section Manufacturing Requirements, must be able to be traced to processes and physical resources on the shop floor which indicate the use of AP239 PLCS. These two standards, AP233 System Engineering and AP239 PLCS, have been developed in cooperation and are compatible in the areas where they overlap. Since the standards are harmonized they contain the same basic requirement representation.

Although tracing of requirements can only be made between requirements (ISO 10303-1142, 2004) cf. Figure 3, AP233 System Engineering and AP239 PLCS both contain a mechanism which can be extended to specify the entities that requirements may originate from. The entity "requirement_source" (ISO 10303-1233, 2004) uses an extensible select "requirement_source_item" to point to the data type that is the source of the requirement, see Figure 2. The figure has been simplified and does not include the entire information structure for a requirement. The "requirement_view_definition" is a view of the requirement that is valid within a specified context. It is at the view definition level that the requirement_source is represented.

Figure 3 Representation of manufacturing requirements in AP233 and AP239.

\section{Conclusion}

The study showed that it is possible to achieve a higher degree of accuracy during decision-making when limitations of existing manufacturing processes or equipment are known in early phases of product development. But to get a full change impact evaluation all aspects must be known. Access to all relevant information requires a lifecycle representation that includes information about products, processes and resources.

The following hypotheses were stated:

Complete and integrated information structures of the product, process, and resource domain is a prerequisite for performing a change impact evaluation.

Requirement information regarding the different domains and the relationships between them is necessary in order to make informed decisions based on the impact evaluation.

The research shows that both AP233 System Engineering and AP239 PLCS may be used for manufacturing requirements representation. However AP233 System Engineering has a richer requirement representation. 
AP233 System Engineering enables full property-based requirement representation while AP239 PLCS has limited support for requirements. This indicates that AP233 System Engineering is more suitable for the manufacturing requirement representation presented in this research article.

\subsection{Verification of Manufacturing Requirements}

In traditional requirement management, a requirement is realized by an aspect of a product or a system. The situation for manufacturing requirements requires that a requirement may be met by an aspect of a process or a manufacturing resource as well. Because of the complexity of the situation it is a difficult task to evaluate and verify manufacturing requirements. This puts even higher demands on the representation of the requirements in order to simplify the verification process.

When evaluating if a requirement is met or not, the ability of a process or resource is matched against the requirement. When using text-based requirement, this becomes a manual activity without any possibility of being automated. The desired situation is to have a model-based requirement description and automatically evaluate if the requirements are met. A geometry definition of the product with features and tolerances will then be possible to match against a similar model of the resource and the process.

\section{Model-based Requirements}

This introduces the idea of model-based requirements where the model itself or part of it defines the requirement. Both the model and the requirement will have different versions at different points in time. Through the use of the model based requirement representation it is then possible to have full traceability of the requirement. A specific version of the requirement will be explicitly linked to a specific version of the model. Hence, when the model is updated it will trigger an update of the requirement, e.g. the resource is upgraded and the ability changes, which in turn changes the manufacturing requirement.

The model-based approach enables different types of characteristics to be used as requirements. A characteristic may be a property, feature, tolerance, or any other relevant concept. For example, geometry models of the resource may be used to state limitations on the size of the part to be machined in the resource.

Figure 4 shows an illustration of the type of information contained in a product model. There is geometry, features, and different types of tolerances.

Figure 4 A geometry model with tolerances.

The model in Figure 4 may also serve as a useful example of model-based requirements for a manufacturing process.

A process plan for the machining of the part may include several operations. Each operation have different geometries of the product model identified as a requirement. For instance:

- Operation 1 may be the machining of the top face. The requirement would then include the desired surface finish as specified for that face in the product model. This requirement must be met by the process, which implies that the machine performing the process must be able to machine to the desired surface finish. 
- Operation $\mathrm{N}$ is the machining of the holes facing forward. The requirement would in this case be a hole pattern, including a feature definition, the pattern (three in a row), the hole tolerance, and the relative position tolerance of the holes.

Depending on the representation of the necessary information in the product model, the requirement definition will look a bit different.

The desired representation is a model that makes use of feature definitions and geometric dimensioning and tolerancing, as is possible using AP203ed2 and AP224. (ISO 10303-203, 2005; ISO 10303-224, 2001)

It would then be possible to simply create a relationship to the feature pattern as the definition of the requirement. The feature pattern will contain all of the desired information in the hole example above.

If the representation of the geometry in the product definition is less intelligent, the definition of the requirement will require more relationships to be defined, but it will still be possible to use the overall approach.

\subsection{Model-based Requirements in AP239 PLCS and AP233 System Engineering}

A good starting point for a representation for model-based manufacturing requirements is AP233 System Engineering. As stated earlier in Section Conclusion, AP233 System Engineering only goes as far as propertybased requirement representation. If the model-based approach is going to work, it is necessary to be able to use objects in the geometry definition as sources for the requirements. However, AP233 System Engineering as well as AP239 PLCS, are currently limited by the fact that they do not include a geometry definition. A representation similar to that of AP214 (ISO 10303-214, 2003), which includes the geometry definition, is necessary to be able to point "in to" the geometry model and identify individual geometry objects. Although neither AP233 System Engineering, nor AP239 PLCS, includes geometry representation by themselves, both have a mechanism that makes it possible to link to a geometry model, e.g. AP214 or AP203. Whether or not this is sufficient has not been verified yet.

\section{Discussion}

Model-based requirements as presented in this research article would be beneficial to manufacturing requirement management, but this approach is far from the way requirements are managed today.

A common view regarding property-based requirements is that they are difficult to create; it is difficult to quantify the requirement in a property that may be automatically evaluated.

Using the model-based approach presented here may alleviate some of this difficulty but will introduce others, e.g. version management of requirements based on parts of a geometry definition.

Furthermore, the problem with defining property-based requirements may stem from the fact that the quantification highlights the fact that the requirement is not stated clearly enough, or not stated in an unambiguous way. 


\subsection{Does the Model-based Approach Eliminate the Need for Requirements?}

It may be argued that the need for requirements is eliminated if a part of the model defines the requirement instead of stating the requirement separately. It is important to realize that the requirement is very much still needed.

The requirement will identify which part of the model is relevant as a requirement in a given context. Furthermore, it is also possible to represent different levels of importance of the requirements when using an explicit requirement representation.

Without the requirement representation, it is also not possible to represent breakdowns of requirement structures or to classify the requirements.

The defining information content of the requirement will be derived from the product, process or resource model, but the requirement itself is still necessary.

\subsection{Reference Data Libraries}

AP233 System Engineering is a very generic model which uses reference data to classify the information content in order to have an explicit information representation.

The reference data necessary for the management of manufacturing requirements will have to be created in order to use AP233 System Engineering for the purpose of manufacturing requirement representation.

An additional benefit of the use of reference data is the strict use of terminology when defining the requirements. As stated by (Kar \& Bailey, 1996), the vocabulary used when defining requirements is important to ensure the correct interpretation of the requirement.

Through the use of reference data, it is possible to restrict the vocabulary and ensure that all requirements are defined using the exact same vocabulary. This is achieved by not entering the information manually, but rather selecting the correct term from the reference data library.

The reference data library is shared by all users, and ensures that everyone uses the same vocabulary.

\subsection{Future Work}

The next step is to test the hypotheses by implementing manufacturing requirement data in the chosen application protocol of STEP. The use of AP233 System Engineering to represent manufacturing requirements will be tested in an ongoing Swedish research project. The representation of manufacturing requirements will also be tested in two different requirement management and PLM-systems. The purpose is to evaluate how the information models may be implemented and what additional support is needed. Communication of these types of requirements between different systems using AP233 System Engineering will also be tested. The work is scheduled to be finished in December 2008. 


\section{Acknowledgements}

This research article is a result of the research conducted in the ModArt (Model driven parts manufacturing) project. ModArt is a collaborative research project with several research organizations and industry partners, which is financed by VINNOVA. The participants include KTH, IVF, Scania, Sandvik Coromant, ITT-Flygt, Haldex Brake Systems, NMW, UGS, Dassault Systèmes, PTC, Eurostep, SIS, Epsilon, and Microtech.

\section{Bibliography}

Bailey, I. (2004). A shared Data Environment for Requirements v1.0. Eurostep Technology White Paper. Eurostep. (2008, April). Manufacturing Life-cycle Seminar. Stockholm, Sweden: Eurostep. Gilb, T. (1997). Towards the Engineering of Requirements. Requirements Engineering (2), 165-169. Harwell, R., Aslaksen, E., Hooks, I., Mengot, R., \& Ptack, K. (1993). What is a Requirement? 3rd International Symposium of the INCOSE.

INCOSE. (2004). Systems Engineering Handbook. International Council On Systems Engineering. ISO 10303-1142. (2004). Industrial Automation Systems and Integration - Product Data Representation and Exchange - Application module: Requirement view definition relationship. International Organization for Standardization.

ISO 10303-1164. (2004). Industrial Automation Systems and Integration - Product Data Representation and Exchange - Application module: Product as individual. International Organization for Standardization. ISO 10303-1233. (2004). Industrial Automation Systems and Integration - Product Data Representation and Exchange - Application module: Requirement assignment. International Organization for Standardization. ISO 10303-203. (2005). Industrial Automation Systems and Integration - Product Data Representation and Exchange - Part 203: Application Protocol: Configuration Controlled Design (Second ed.). International Organization for Standardization.

ISO 10303-214. (2003). Industrial Automation Systems and Integration - Product Data Representation and Exchange - Part 214: Application Protocol: Core Data for Automotive Mechanical Design Processes (Second ed.). International Organization for Standardization.

ISO 10303-224. (2001). Industrial Automation Systems and Integration - Product Data Representation and Exchange - Part 224: Application Protocol: Mechanical Product Definition for Process Planning Using Machining Features. International Organization for Standardization.

ISO 10303-239. (2004). Industrial Automation Systems and Integration - Product Data Representation and Exchange - Part 239: Application Protocol: Product Life Cycle Support. International Organization for Standardization.

ISO CD 10303-233. (2007). Industrial Automation Systems and Integration - Product Data Representation and Exchange - Part 233: Application Protocol: System Engineering and Design. International Organization for Standardization.

Johansson, M. (2001). Information Management for Manufacturing System Development. Kungliga Tekniska Högskolan.

Kar, P., \& Bailey, M. (1996). Characteristics of Good Requirements. Proceeding of the 6th Sixth international symposium of the INCOSE.

Kerr, C. I., Roy, R., \& Sackett, P. J. (2006). Requirements management: An enabler for concurrent engineering in the automotive industry. International Journal of Production Research, 44 (9), 1703-1717.

Kirkman, D. P. (1998). Requirement Decomposition and Traceability. Requirements Engineering , 3 (2), 107 114. 
Lang, M., \& Duggan, J. (2001). A Tool to Support Collaborative Software Requirements Management. Requirements Engineering , 6 (3), 161-172.

Melchisedech, R. (1998). Investigation of Requirements Documents Written in Natural Language. Requirements Engineering (3), 91-97.

Micouin, P. (2008). Toward a Property Based Requirements Theory: System Requirements Structured as a Semilattice. Systems Engineering , 11 (3).

Nielsen, J. (2003). Information Modeling of Manufacturing Processes: Information Requirements for Process Planning in a Concurrent Engineering Environment. Kungliga Tekniska Högskolan.

Nilsson, P. (2004). Managing Requirements in a Design Support System - a Product Modelling approach. Chalmers University of Technology, Sweden.

Nyqvist, O. (2008). Information Management for Cutting Tools: Information Models and Ontologies. Kungliga Tekniska Högskolan.

Ratchev, S., Pawar, K. S., Urwin, E., \& Dirk, M. (2005). Knowledge-enriched Requirement Specification for One-of-a-kind Complex Systems. Concurrent Engineering , 13 (3), 171.

Rogstrand, V., \& Kjellberg, T. (2004). Interaction of Requirements in Manufacturing System Development. Proceedings of the 14th International CIRP Design Seminar.

Rogstrand, V., \& Kjellberg, T. (2005). Representation of Manufacturing Requirements in Collaborative Environments. International Conference on Manufacturing Research. Great Britain: Cranfield University. Selvaraj, P., Radhakrishnan, P., \& Adithan, M. (2008). An integrated approach to design for manufacturing and assembly based on reduction of product development time and cost. International Journal of Advanced Manufacturing Technology .

Stevens, R., Jackson, K., Brook, P., \& Arnold, S. (1998). Systems Engineering Coping with Complexity. Hertfordshire, UK: Prentice Hall Europe.

Ulrich, K. T., \& Eppinger, S. D. (2000). Product Design and Development (Second ed.). McGraw-Hill. Yin, R. K. (1990). Case Study Research - Design and Method. Sage Publications. 